\title{
Groupwise Morphometric Analysis Based on High Dimensional Clustering
}

\author{
Dong Hye Ye ${ }^{1, *}$, Kilian M. Pohl ${ }^{2}$, Harold Litt ${ }^{1}$, and Christos Davatzikos ${ }^{1}$ \\ ${ }^{1}$ Department of Radiology, University of Pennsylvania, Philadelphia, PA, USA, 19104 \\ ${ }^{2}$ IBM Almaden Research Center, San Jose, CA, USA, 95120
}

\begin{abstract}
In this paper, we propose an efficient groupwise morphometric analysis to characterize morphological variations between healthy and pathological states. The proposed framework extends the work of Baloch [4] in which a manifold for each anatomy was constructed by collecting lossless [transformation, residual] descriptors with various transformation parameters, and the optimal set of transformation parameters was estimated individually by minimizing group variance. However, full parameter exploration is not desired as it can result in transformation leading to inaccurate anatomical models. In addition, a single fixed template introduces a priori bias to subsequent statistical analysis. In order to overcome these limitations, we use an affinity propagation clustering method to find the spatially close cluster center for each subject. Then, a subject is normalized to the template via the cluster center to restrict our analysis only to those descriptors that reflect reasonable warps. In addition, a mean template is selected by finding a cluster center that minimizes the sum of pairwise shape distance to reduce the fixed template bias. Our method is applied to $2 D$ synthetic data and $3 D$ real Cardiac MR Images. Experimental results show improvement in quantifying and localizing shape changes.
\end{abstract}

\section{Introduction}

Computational anatomy is a widely used method for quantifying and locating morphological changes between healthy and pathological structures. Most studies in computational anatomy, such as [2], [7], [1], [8], use deformable registration from subjects to a template. The resulting transformations from subjects to a template are analyzed for inter-subject comparisons. These approaches assume that a perfect transformation captures its morphological characteristics relative to a template. Such transformations are driven by solving optimization problem which maximizes the im- age similarity measures (i.e. intensity difference or mutual information) between a template and the warped subject. Typically, smoothness constraints are imposed on transformation to preserve topological relationship among anatomical structures.

However, in practice, it is difficult to find a perfect transformation due to inherent complexity of the problem. First, anatomical correspondence may not be uniquely determined from intensity-based image attributes. For example, different cardiac muscles such as the myocardium or papillary muscle in an MRI have similar intensities. Second, exact anatomical correspondence may not exist at all due to anatomical variability across subjects. For instance, in the brain, it may not be possible to perfectly warp a double-folded cingulate sulcus to a single-folded cingulate sulcus through a biologically meaningful transformation, as illustrated in Fig. 1. As a result, the choice of a template plays an important role in the accuracy of the analysis. Anatomies closer to the template are well represented by a diffeomorphism. However, large differences between an individual and the template lead to residual information that the transformation does not capture. Therefore, a priori fixed template introduces a bias to subsequent statistical analysis.

Some approaches [5], [22], [9] have recently been proposed to remedy this template bias problem in computational anatomy. In Lepore's work [16], each individual image is registered to multiple templates and average deformation from multiple registrations were computed for each image. In Sabuncu's work [18], Bayesian scheme was used to discover modes in an image population. Even though these approaches solve the priori template bias problem to a certain extent, they fail to deal with variation from registration parameters.

Generally, registration algorithms depend on two transformation parameters: the intermediate template and the smoothness level of transformation. When a subject is mapped to the largely different template, the overall registration between the template and the subject can be obtained 


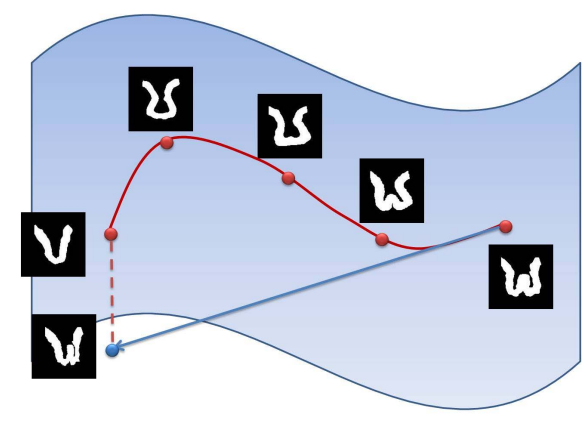

Figure 1: The figure illustrates the difficulty of registration between single-folded cingulate sulcus and double-folded cingulate sulcus. When the smoothing factor is too small, the direct path of minimization deviates from the space of diffeomorphism and causes unnatural distortion.

by combining the deformation fields that warp the template to the intermediate template, and the intermediate template to the subject. Appropriate selection of intermediate templates can aid registration, because a subject who is not well represented by the template can be registered effectively via spatially closer intermediate template. In addition, the level of regularity largely affects the transformation because it is embedded in cost function of optimization problem as a smoothness constraint. Although one may argue that a very aggressive registration is desirable because it eliminates the residual, this may create biologically implausible correspondences. Aggressive warping may also lead to noisy deformation fields that are unsuitable for subsequent statistical analysis [6]. Differences in the intermediate template and regularization parameter lead to different representations of the same anatomy. Variations of these parameters can be considered as confounding factors. However, both parameters are often arbitrarily or empirically chosen. More importantly, they are not optimized for each anatomy: individually, this leads to an unwanted confounding variation in the morphological measurements, which reduces the ability to detect subtle abnormalities.

To solve this confounding variation problem, Baloch [4] proposed groupwise morphometric analysis. In groupwise morphometric analysis framework, a manifold for each subject is constructed by a complete morphological descriptor (CMD), which is the concatenated vector of transformation and residual; any morphological information not captured by the transformation is captured by the residual, hence no morphological characteristic is discarded. An entire class of many anatomically equivalent descriptors is generated by varying regularization parameter as well as the intermedi- ate templates. The resultant anatomical equivalence class (AEC) maps the underlying anatomy to a manifold embedded in a high dimensional space, which is coined as a morphological appearance manifold (MAM). The optimal morphological signatures (OMS) are obtained by minimizing the variance across MAM.

One limitation of Baloch's work [4] is the redundancy problem. We can represent an individual's anatomy with a biologically implausible transformation of a template, as long as we keep track of the residual that is necessary to construct that manifold. In other words, one can construct a very broad manifold, by including all possible transformations and respective residuals. Although this would be of theoretical interest, in practice it leads to an unnecessarily difficult optimization problem because the optimal representation would be corrupted by biologically implausible representation. To more effectively find the optimal groupwise registration, it is desirable to restrict our analysis only to those pairs [Transformation, Residual] that reflect reasonable warps from one anatomy to another. This is a much more restricted manifold, which is likely to have smooth structure leading to fewer local minima, and allow for the optimal solution to be found much faster as the search space is small.

This paper presents an efficient and effective groupwise morphometric analysis framework to cope with aforementioned issues. To alleviate the redundancy problem, we detect the spatially close exemplars by affinity propagation clustering method. By taking small steps to the nearest shape, one can find a series of intermediate templates leading from one anatomy to another. This allows a compact manifold construction which only includes biologically plausible [Transformation, Residual] pairs. To address the priori fixed template bias, we compute the average shape anatomy through geodesic distance on a learned manifold. By finding the cluster center that minimizes the sum of pairwise shape distances, we can calculate the most representative template which reduces the bias.

In the remainder of this paper, we present our framework in Section 2 and provide experimental validations in Section 3. The paper is concluded in Section 4.

\section{Methods}

Fig. 2 summarizes our groupwise morphometric analysis framework. We assume that given images are linearly aligned [20] and bias field corrected. First, the images are clustered into different classes, each containing only those that are spatially close to each other in their intrinsic highdimensional space. Then, the average shape of cluster centers is selected as an unbiased template. Each subject is normalized to the mean template via cluster centers at different regularization level. This constructs a manifold that includes [Transformation, Residual] descriptor pairs. After 
Preprocessing:

Linear alignment, bias field correction

Affinity Propagation Clustering:

Find spatially close cluster center

Mean shape template:

Select the average shape anatomy

MAM optimization:

Estimate optimal morphological descriptors

Voxel-wise statistical test:

Hotelling $T 2$ test

Figure 2: Overview of proposed framework

minimizing group variance, we find the optimal representation which best highlights the group differences. Hotelling $T^{2}$ test is performed for voxel-wise statistical test.

\subsection{Morphological Appearance Manifold}

Computational anatomy characterizes anatomical differences between a subject $S$ and a template $T$ by analyzing transformation $h: \Omega_{T} \rightarrow \Omega_{S}$. It assumes that there exists a conforming spatial transformation which perfectly carries morphological characteristic without residual. But, a zero residual mapping is usually very difficult to find, resulting in a residual,

$$
R_{h}(\mathbf{x}):=T(\mathbf{x})-S(h(\mathbf{x})), \mathbf{x} \in \Omega_{T} .
$$

Since some anatomical characteristics not captured by transformation reside in residual image, subsequent analysis cannot detect morphological variations perfectly if only transformation properties are examined. To avoid the loss of descriptor, our approach herein uses a complete morphological descriptor (CMD), which combines transformation properties with the respective residual. The transformation and the residual were combined into a concatenated descriptor

$$
\mathcal{Q}_{h}(\mathbf{x}):=\left(J_{h}, R_{h}\right)
$$

where $J_{h}$ is the Jacobian determinant.

However, $\mathcal{Q}_{h}$ depends not only on the underlying anatomy but also on transformation parameters. The choice of transformation parameters leads to an unwanted confounding variation, which may interrupt the detection of subtle morphological variations. Therefore, we need to minimize the variation from transformation parameters. In order to minimize the variation, we construct an anatomical equivalence class (AEC)

$$
\begin{array}{r}
\mathcal{A}(S)=\left\{\mathcal{Q}_{h_{\theta}}(\mathbf{x}): S\left(h_{\theta}(\mathbf{x})\right)=T(\mathbf{x})-R_{h_{\theta}}(\mathbf{x}),\right. \\
\left.\forall \mathbf{x} \in \Omega_{T}, \forall \theta \in \mathbf{\Theta}\right\},
\end{array}
$$

where $\theta$ is the transformation parameter. AEC is the collection of $\mathcal{Q}_{h}$ that reconstructs the same subject

All AECs construct a manifold which is called morphological appearance manifold (MAM). Because there are infinitely many ways in which the same anatomy can be represented via [Transformation, Residual] pairs, we need to determine the optimal position on each anatomy by solving an optimization problem that minimizes group variance. In order to minimize group variances, we should find representations that yield minimum pairwise distance between AECs. These representations best highlight the morphological variation, because they have similar amount of variations from transformation parameters and thus eliminate the variation when comparing descriptors.

Since [Transformation, Residual] pairs are only dependent on the transformation parameter $\theta_{S_{i}}$ for a given subject $S_{i}$, we drop the subscript to represent AECs as $\mathcal{A}^{i}\left(\theta_{i}\right)$. The distance between two AECs can be defined by the closest subspace pair as follows [17]:

$$
d\left(\mathcal{A}^{i}\left(\theta_{i}\right), \mathcal{A}^{j}\left(\theta_{j}\right)\right)=\min _{C_{i} \in \mathcal{A}^{i}\left(\theta_{i}\right), C_{j} \in \mathcal{A}^{j}\left(\theta_{j}\right)} d\left(C_{i}, C_{j}\right),
$$

where $C_{i}$ and $C_{j}$ are the local linear subspaces. Clearly, the similarity between two AECs is computed as the similarity between their best suited local models.

We find the point minimizing the pairwise sum of AEC distance for $L$ anatomies.

$$
\boldsymbol{\Theta}^{*}=\arg \min _{\Theta} \sum_{i} \sum_{j} d\left(\mathcal{A}^{i}\left(\theta_{i}\right), \mathcal{A}^{j}\left(\theta_{j}\right)\right),
$$

where $\boldsymbol{\Theta}=\left(\theta_{1}, \theta_{2}, \ldots, \theta_{L}\right)$. It provides optimal combination of transformation and residual by minimal variance criterion.

For transformation parameters, we concentrate on intermediate template $\tau \in \mathcal{T}$ with $\mathcal{T}$ representing all possible templates and regularity of deformation field $\lambda \in \mathbb{R}_{+}$. Since analysis eventually has to be carried out in a common space, we ultimately bring all anatomies to the template space $\Omega_{T}$. However, intermediate templates capture the variation we expect to see when the same anatomy is seen via different templates. Variations of the regularization parameter $\lambda$ reflect variations observed by varying the degree of conformity of the transformation and large residuals. At $\lambda=0$, the most aggressive transformation of the template under the assumptions of a respective deformable registration algorithm seeks to minimize the residual.

Then, AEC is generated by registering each subject via 


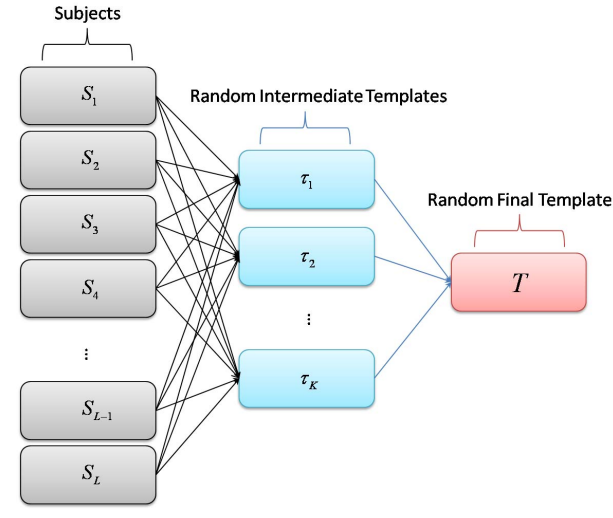

(a)

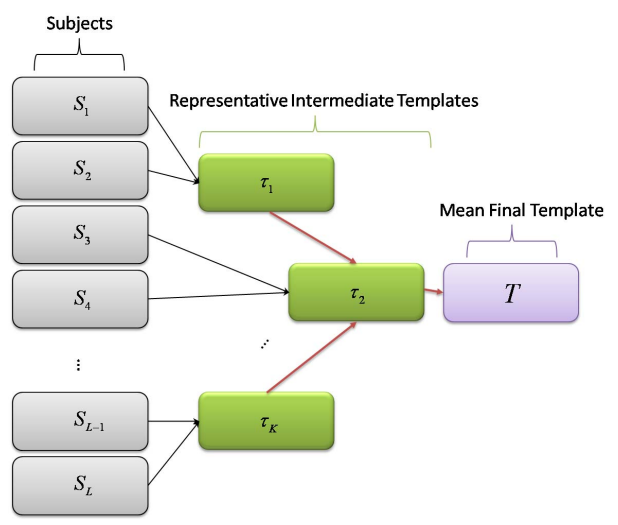

(b)

Figure 3: Constructing AECs: (a) Each subject is normalized to the typical final template via random intermediate templates. (b) Each subject is first registered onto a representative template and then mapped to the mean final template

random intermediate templates at different regularization level, as shown in Fig. 3(a). But, constructing AEC with all possible intermediate templates leads to unnecessarily difficult optimization problem, because intermediate template largely different from the template and subject introduces distorted deformation. So, it is desirable to restrict our analysis only to [Transformation, Residual] pairs that reflect reasonable warps from one anatomy to another. Instead of using random intermediate templates, we use the intermediate template which is spatially close to a subject. Fig. 3(b) shows our method of constructing AECs. Each subject is mapped to the final template via the intermediate template with similar shape.

Our AEC construction requires much less registrations. Suppose that we have $L$ subjects, $K$ intermediate templates, and $\Lambda$ regularization parameters. For conventional AEC construction, $\Lambda \times(L \times K+K)$ registrations need to be performed. On the contrary, our AEC construction requires only $\Lambda \times(L+K)$ registration. Furthermore, this is a much more restricted manifold, which is likely to have smooth structure leading to fewer local minima and allow for the optimal solution to be found much faster as the search space is small.

\subsection{Affinity Propagation Clustering}

In order to find a spatially close intermediate template for each subject, we apply an affinity propagation clustering method [10] to the data. In contrast to the conventional $k$ mean clustering techniques, affinity propagation considers all images as potential exemplars without random initial selection of cluster center. Therefore, the number of classes and initial cluster centers are not manually set before clustering.

It views each image as a node in a network and each image communicates the messages along the edges of the network. There are two kinds of messages exchanged between data points. The "responsibility" $r(i, k)$ is sent from data $i$ to cluster $k$, which reflects the likelihood that data $i$ belongs to center $k$. The "availability" $a(i, k)$ is sent from cluster $k$ to data $i$, which reflects the likelihood that cluster $k$ serves as the exemplar for data $i$. For responsibility, the real-valued similarity measure $s(i, k)$ between each pair of images should be given. Then, the responsibility $r(i, k)$ and the availability $a(i, k)$ are updated by the rules:

$$
\begin{gathered}
r(i, k) \leftarrow s(i, k)-\max _{k^{\prime} s . t . k^{\prime} \neq k} a\left(i, k^{\prime}\right)+s\left(i, k^{\prime}\right) ; \\
a(i, k) \leftarrow \min (0, r(k, k))+\sum_{i^{\prime} s . t . i^{\prime} \notin i, k} \max \left(0, r\left(i^{\prime}, k\right)\right) .
\end{gathered}
$$

In order to apply affinity propagation method to image dataset, we first need to define the similarity measure between each pair of images. Since we want to cluster the images which have similar shape, mutual information can be used as an approximation of shape distance. Furthermore, for the dataset which has been linearly aligned and bias field corrected, mutual information (MI) is sufficient because overlapping regions of two images are relatively large. Therefore, we have adopted MI as the similarity measure.

Besides the measurement of similarity between each pair of images, affinity propagation requires the "self similarity" or "preferences" $s(k, k)$ for data $k$. These values represent prior probability for a certain image to be an exemplar in a detected class. Since all data points are equally suitable as exemplars, we set a common preference value to all images. The common preference value can range from the minimum to the median of the input similarities. It has been shown that the median preference value results in a moderate num- 


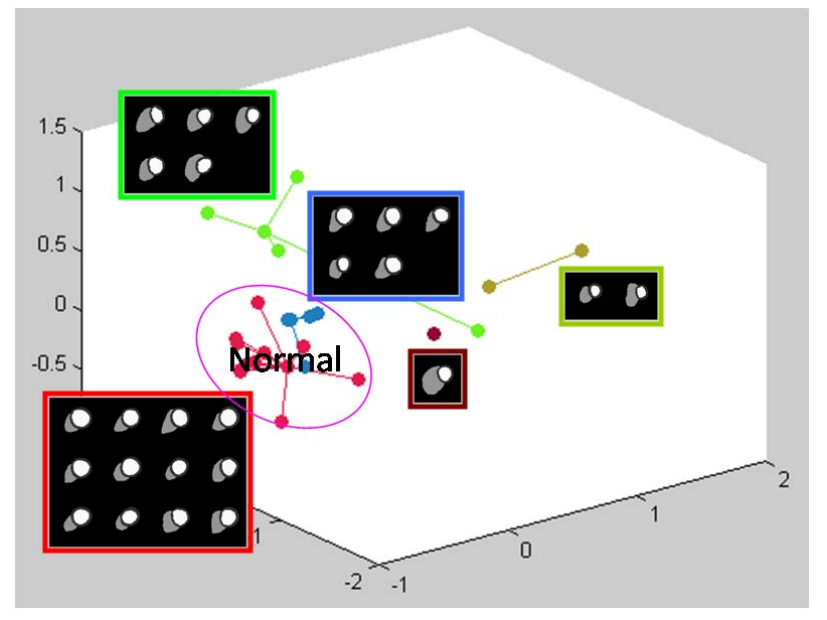

Figure 4: Example of affinity propagation clustering on 3D Cardiac MRI

ber of clusters and the minimum one results in a small number of clusters. Since the size of cluster should not be large, we select the median of input pairwise similarity measures as preference.

Fig. 4 shows the example of clustering obtained using the affinity propagation method on 3D cardiac MR Images. Since these shape manifolds are embedded in a very highdimensional space, we calculated the first three principal components for visualization purposes. The images are clustered into different classes, each containing only images that are spatially close to each other in their intrinsic highdimensional space.

\subsection{Geodesic Mean Template}

After exemplars are selected, each subject is registered to the single fixed template. Even though these exemplars help registration, the final template bias still exists. So, it is necessary to reduce the final template bias for better morphometric analysis. Geodesic registration approach [3], [14] has provided a mathematically elegant solution for this unbiased template selection problem. An unbiased template of the given data can be defined as the geodesic mean of the data. But, the numerical computation of the velocity fields is quite time-consuming which can outweigh its benefits. To find the geodesic mean template efficiently, we approximate the geodesics by composing the multiple small deformations observed in the actual anatomies in the data and find the pseudo geodesic mean template from approximated geodesics.

In order to approximate geodesics, we formulate the geodesic distance of two different images. A diffeomorphic coordinate transformation between two image is $h(x)=$ $\phi(x, 1)$, where $\phi(x, t)=x+\int_{0}^{t} v(\phi(x, \tau), \tau) d \tau$, and $v(x, \tau)$ is a smooth time varying vector field. The diffeomorphic framework includes a metric on the diffeomorphic transformation $\|\phi(x, t)\|_{L}=\int_{0}^{t}\|v(x, \tau)\|_{L} d \tau$, which induces the metric between image $I_{i}$ and $I_{j}$ :

$$
\begin{aligned}
& \qquad d\left(I_{i}, I_{j}\right)=\min _{v} \int_{0}^{1}\|v(x, \tau)\|_{L} d \tau \\
& \text { subject to } \int_{\Omega}\left\|I_{i}(x+\phi(x, 1))-I_{j}(x)\right\|_{2}^{2} d x=0 .
\end{aligned}
$$

The metric prioritizes the mappings and, with an appropriate choice of the differential operator $L$ in the metric, ensures smoothness. We introduce the constraint that the transformation must provide a match between the two images. For two images that are very similar, $\phi$ and $v$ are small, and because the velocities of the geodesics are smooth in time, we can approximate the integrals for the coordinate transform and geodesic distance:

$$
\begin{aligned}
& \qquad d\left(I_{i}, I_{j}\right)=\min _{v}\|h(x)\|_{L} \\
& \text { subject to } \int_{\Omega}\left\|I_{i}(x+h(x))-I_{j}(x)\right\|_{2}^{2} d x<\epsilon,
\end{aligned}
$$

where $\epsilon$ is the noise of the image.

Since two images are very similar, we can further approximate the geodesic distance by using Lagrangian multiplier, yielding

$d\left(I_{i}, I_{j}\right)=w \int_{\Omega}\left\|I_{i}(x+h(x))-I_{j}(x)\right\|_{2}^{2} d x+\int_{\Omega}\|h(x)\|_{L} d x$,

where $w$ is the weight. Here, we use the operator $L=\nabla$ and $\|h(x)\|_{L}=\|\nabla h(x)\|_{2}$. Then, geodesic distance for similar two images can be approximated to weighted sum of mean squared error and harmonic energy [12], [11]. In this paper, we empirically set the weight to 0.5 .

For largely different images, the geodesic distance can be approximated by taking small steps to the nearest. We replace the shortest path of the analytical manifold to the shortest path on a k-nearest-neighbor $(k \mathrm{NN})$ graph that approximates the metric structure. The edge of the graph is assigned a weight equal to the distance $d\left(I_{i}, I_{j}\right)$ between two similar images. Then geodesic distance between two arbitrary images can be approximated by the sum of its edge length along the path

$$
g\left(I_{i}, I_{j}\right)=\sum_{(k, l) \in \mathcal{A}_{i, j}} d\left(I_{k}, I_{l}\right),
$$

where $\mathcal{A}_{i, j}$ is the shortest path in $k \mathrm{NN}$ graph from $I_{i}$ to $I_{j}$.

Then, we can find the geodesic mean template by using $g\left(I_{i}, I_{j}\right)$. We choose the geodesic median template as the final template since it is unbiased:

$$
I_{T}=\arg \min _{i} \sum_{j} g\left(I_{i}, I_{j}\right)
$$




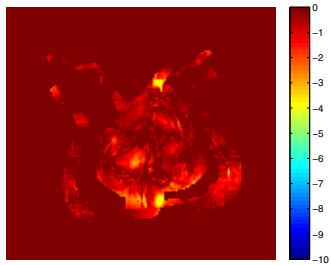

(a)

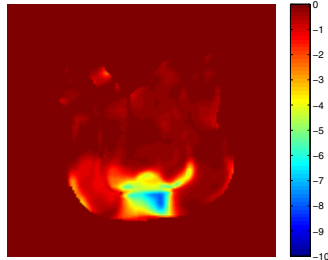

(b)

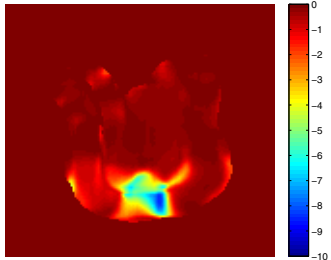

(c)

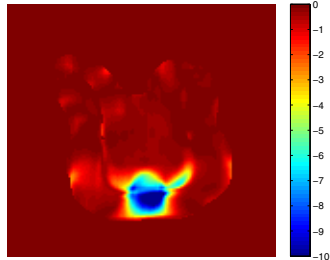

(d)

Figure 5: $p$ value maps for 2D synthetic brain patch (a) worst CMD at $\lambda=1$ (b) best CMD at $\lambda=4$ (c) OMS with random intermediate templates (d) OMS with clustering

The most time-consuming part in practice is the pairwise registration between all images which requires $O\left(n^{2}\right)$ registrations. Suppose that there are $n$ images, $n(n-1) / 2$ pairwise registrations need to be performed. But, since we only need to perform pairwise registration between small number of representative images chosen by affinity clustering, we can reduce the overhead. Moreover, we can distribute the registration tasks on a cluster server of multiple CPUs, since registration of one pair is independent of the other pairs.

\section{Experimental Result}

To evaluate the proposed morphometric analysis method, we apply it to two datasets. The first is a group of $2 \mathrm{D}$ simulated brain patches and the second is 3D real Cardiac MR images. For non-rigid registration, we use the diffeomorphic demons [19] due to its efficiency. Images were registered with various smoothing kernel sizes from one to four which indicates the level of regularity.

\subsection{D simulated brain patch}

A 2D dataset of 60 shapes was generated by introducing random variability in 12 manually created templates. Thinning was introduced in center of the fold of 30 subjects to simulate atrophy.

First, we compare the level of significance of group differences based on various values of the smoothing parameter $\sigma$, the standard deviation of the Gaussian filter used to smooth the residual prior to the statistical test. We plot the minimum logarithm of $p$ value maps as the function of $\sigma$ in Fig. 6 to indicate the best achievable performance. It is observed that aggressive registration $(\lambda=1)$ shows the worst performance. This observation is in accordance with our hypothesis that overly aggressive transformation contaminates statistical analysis. The best performance of CMD is achieved with moderate transformation at $\lambda=4$ $\left(p=10^{-12.7}\right)$. After MAM optimization, the absolute minimum $p$ value improves to $10^{-13.4}$, which demonstrates that OMS slightly outperforms the best possible CMD. In proposed framework, OMS $\left(p=10^{-15.9}\right)$ significantly outperforms the CMD with the help of clustering.

It should be noted that the minimum $p$ value plots do not completely represent spatial distribution. In order to compare the localization of group difference, $p$ value maps are displayed in Fig. 5 at $\sigma=6$. The worst $\operatorname{CMD}(\lambda=1)$ fails to accurately localize the atrophy with large numbers of false positive. Regular OMS localizes slightly better than the best CMD. OMS with clustering locates atrophy more precisely, which is in accordance with the location of simulated thinning.

\subsection{D real cardiac MRI}

Tetralogy of Fallot (TOF) [15] is a common congenital heart disease which involves primarily right ventricular abnormalities including right ventricular hypertrophy and right ventricular outflow tract obstruction (RVOT). In order to characterize abnormality of TOF patients in right ventri-

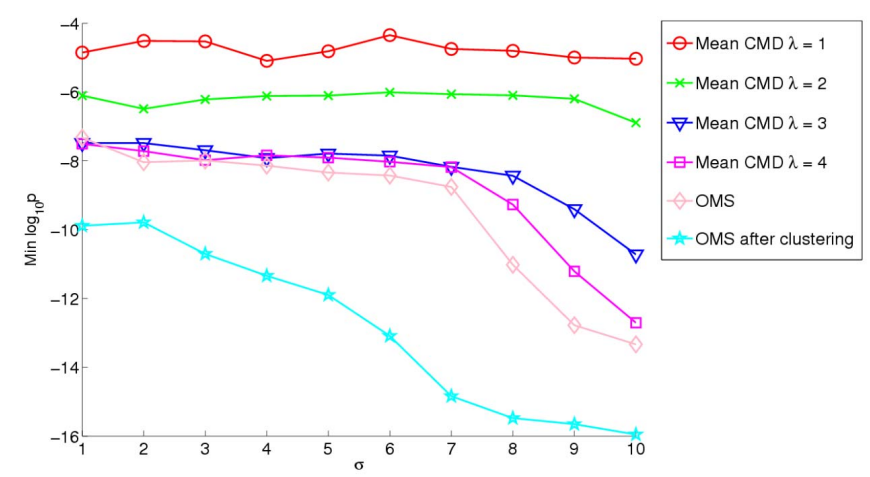

Figure 6: Minimum $p$ value plot for simulated 2D brain patches 


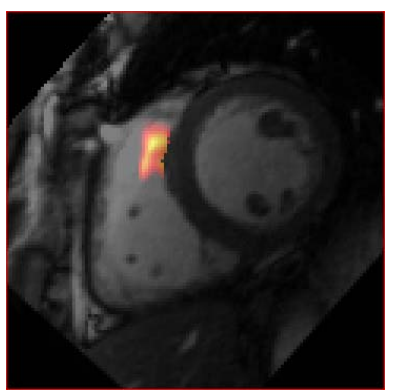

(a)

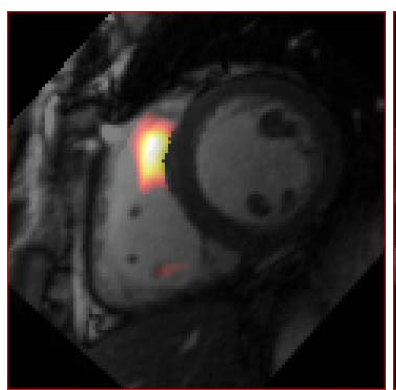

(b)

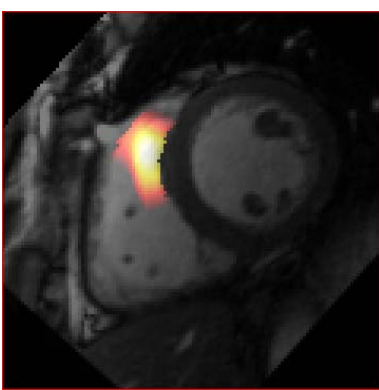

(c)

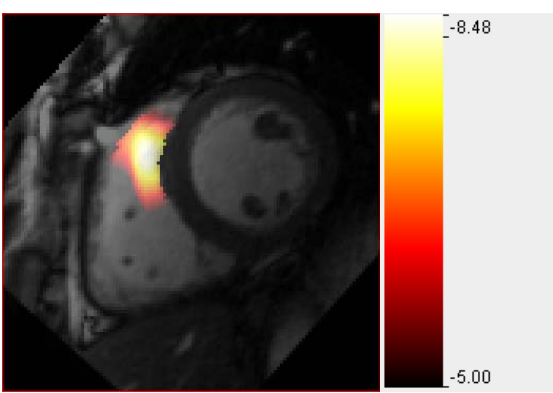

(d)

Figure 7: $p$ value maps thresholded to $p \leq 10^{-5}$ for 3D cardiac MRI (a) worst CMD at $\lambda=1$ (b) best CMD at $\lambda=4$ (c) OMS with random intermediate templates (d) OMS with clustering

cle (RV), steady-state free precession cardiac MR images were obtained at end-diastole in 12 TOF patients and 12 normal volunteers. In addition, we obtained the Auckland Atlas as a typical template [21]. The in-plane resolution was $2 \mathrm{~mm}$ and the slice thickness was $8 \mathrm{~mm}$. The acquired Short Axis (SA) slices covered the whole heart. To prevent other organs from influencing analysis, the CMR data were cropped using manually defined rectangular regions that enclosed the complete left ventricle (LV) and RV on the short axis, respectively. The datasets were semi-automatically segmented on short-axis slices using "Segment" [13]. Three ventricular borders were traced: LV endocardium, LV epicardium, and RV epicardium. The papillary muscles were included in the LV blood pool enclosed by the LV endocardial border.

First, we evaluate the bias of final template. We compare the typical final template (Auckland Atlas) with pseudo geodesic mean template. The minimum logarithm of $p$ value as a function of $\sigma$ is plotted in Fig. 8. For the same regularization parameter $\lambda$, the geodesic mean template yields better performance with about an order of magnitude decrease in $p$ values over typical Auckland Atlas. This im-

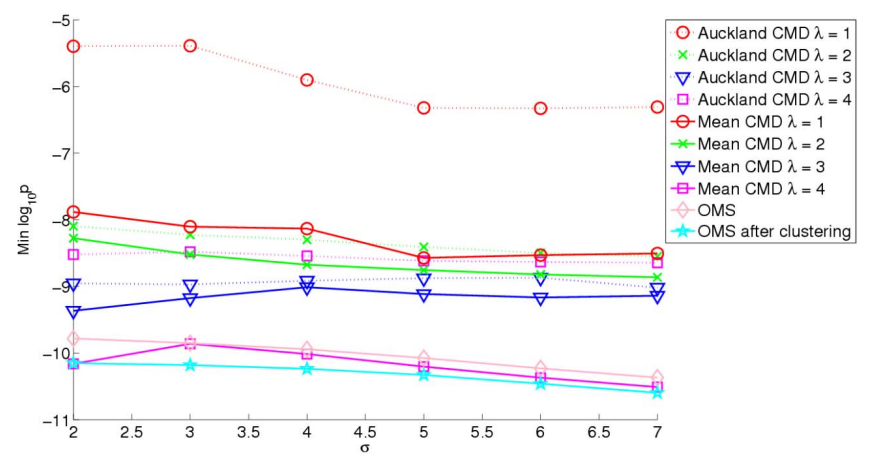

Figure 8: Minimum $p$ value plot for 3D cardiac MRI plies that a fixed template bias is reduced by using geodesic mean shape. In addition, we compare the level of significance of group differences based on CMDs and OMSs. Like 2D synthetic experiment, aggressive registration shows the worst CMD performance at $\lambda=1\left(p=10^{-8.57}\right)$ and conforming registration shows the best CMD performance at $\lambda=4$. But, after optimization with random intermediate templates, the absolute minimum $p$ value increases from $3 \times 10^{-11}$ to $4.2 \times 10^{-11}$. In other words, regular OMS is slightly worse than the best CMD. This demonstrates that broad AEC constructions may corrupt the statistical analysis. On the contrary, after optimization with clustering, the absolute minimum $p$ value improves to $2.5 \times 10^{-11}$. This shows that a compact AEC construction via clustering helps improve statistical power. It is worthwhile noting that in practice we do not know the best value of $\lambda$. Therefore, even though regular OMS shows worse performance than the best CMD, the OMS is clearly better in terms of robustness.

In order to estimate localization, it is essential to threshold small $p$ values in the entire ROI. Therefore, $p$ significant ROIs $\left(p \leq 10^{-5}\right)$ are displayed for CMDs and OMSs corresponding to the $\sigma$ parameter selection that yielded the best performance in Fig. 7. Overall, the RVOT region is characterized as an area of pathology. Note how OMS helps in precisely localizing the RVOT obstruction (Fig. 7 (c) and (d)). On the other hand, CMD with aggressive transformation (Fig. 7 (a)) fails to accurately localize shape abnormalities, producing false positives. The CMD that yields the best performance localized the shape variation relatively accurately (Fig. 7 (b)). But, the OMS is clearly better than CMD if one considers anatomical location of RVOT.

\section{Conclusion}

In this paper, we proposed an efficient and effective groupwise morphometric analysis method for characterizing shape variations by incorporating affinity propagation 
clustering and pseudo geodesic mean template selection into the work in [4]. By affinity propagation clustering, spatially close shapes were clustered to one representative subject efficiently. Over cluster centers, we found the geodesic mean template via learned manifold from real data. These clustering and geodesic mean template play an important role in restricting manifold to reasonable warps and removing the fixed template bias. Then, lossless descriptor [Transformation, Residual] for each anatomy is optimized by minimum group variance criteria. This optimization of transformation parameters can reduce the confounding variation and help improve statistical power. This is confirmed in our experiments on 2D synthetic brain patch and 3D real Cardiac MRI, where our optimization resulted in improvements in quantifying and localizing shape changes over conventional computational anatomy method.

\section{Acknowledgment}

The research was partly funded by an ARRA supplement to NIH NCRR (P41 RR13218).

\section{References}

[1] J. Ashburner and K. J. Friston. Voxel-based morphometrythe methods. NeuroImage, 11(6):805-821, 2000. 1

[2] J. Ashburner, C. Hutton, R. Frackowiak, I. Johnsrude, C. Price, and K. Friston. Identifying global anatomical differences: deformation-based morphometry. Human Brain Mapping, 6(6):348-357, 1998. 1

[3] B. Avants and J. C. Jee. Geodesic estimation for large deformation anatomical shape averaging and interpolation. $\mathrm{Neu}$ roImage, 23:139-150, 2004. 5

[4] S. Baloch and C. Davatzikos. Morphological appearance manifolds in computational anatomy: Groupwise registration and morphological analaysis. NeuroImage, 12(1), 2008. $1,2,8$

[5] K. Bhatia, J. Hajnal, B. Puri, A. Edwards, and D. Rueckert. Consistent group wise nonrigid registration for atlas construction. ISBI, pages 908-911, 2004. 1

[6] P. Cachier. How to trade off between regularization and image similarity in nonrigid registration? MICCAI, pages 1285-1286, 2001. 2

[7] D. Collins, T. Paus, A. Zijdenbos, K. Worsley, J. Blumenthal, J. Giedd, J. Rapoport, and A. Evans. Age related changes in the shape of temporal and frontal lobes: an mri study of children and adolescents. Society Neuroscience Abstract, 1998. 1

[8] C. Davatzikos, A. Genc, D. Xu, and S. M. Resnick. Voxelbased morphometry using the ravens maps: Methods and validation using simulated longitudinal atrophy. NeuroImage, 14:1361-1369, 2001. 1

[9] B. Davis, P. Lorenzen, and S. Joshi. Large deformation minimum mean squared error template estimation for computational anatomy. ISBI, pages 173-176, 2004. 1

[10] B. J. Frey and D. Dueck. Clustering by passing messages between data points. Science, 315:972-976, 2007. 4
[11] S. Gerber, T. Tasdizen, S. Joshi, and R. Whitaker. On the manifold structure of the space of brain. MICCAI, 2009. 5

[12] J. Hamm, C. Davatzikos, and R. Verma. Efficient large deformation registration via geodesics on a learned manifold of images. MICCAI, 12(1), 2009. 5

[13] E. Heiberg, J. Sjogren, M. Ugander, M. Carlsson, H. Engblom, and H. Arheden. Design and validation of segment freely available software for cardiovascular image analysis. BMC Medical Imaging, 10(1), 2010. 7

[14] S. Joshi, B. Davis, M. Jomier, and G. Gerig. Unbiased diffeomorphic atlas construction for computational anatomy. $\mathrm{Neu}$ rolmage, 23, 2004. 5

[15] A. L. Knauth, K. Gauvreau, A. J. Powell, M. J. Landzberg, E. P. Walsh, J, E, Lock, P. J. del Nido, and T. Geva. Ventricular size and function assessed by cardiac mri predict major adverse clinical outcomes late after tetralogy of fallot repair. Heart, 94(2):211-216, 2008. 6

[16] N. Lepore, C. Brun, Y.-Y. Chou, A. D. Lee, M. Barysheva, G. I. de Zubicaray, M. Meredith, K. L. McMahon, M. J. Wright, A. W. Toga, and P. M. Thompson. Multi-atlas tensorbased morphometry and its application to a genetic study of 92 twins. MFCA, 2008. 1

[17] N. Lian and C. Davatzikos. Groupwise morphometric analysis based on morphological appearance manifold. MMBIA, 2009. 3

[18] M. R. Sabuncu, S. K. Balci, and P. Golland. Discovering modes of an image population through mixture modeling. MICCAI, 2008. 1

[19] T. Vercauteren, X. Pennec, A. Perchant, and N. Ayache. Diffeomorphic demons: Efficient non-parametric image registration. NeuroImage, 45(1), 2009. 6

[20] M. Woolrich, S. Jbabdi, B. Patenaude, M. Chappel, S. Makni, T. Behrens, C. Beckmann, M. Jenkinson, and S. Smith. Bayesian analysis of neuroimaging data in fsl. NeuroImage, 45:173-186, 2019. 2

[21] A. Young. The cardiac atlas. 7

[22] L. Zollei, M. Shenton, W. W. III, and K. Pohl. The impact of atlas formation methods on atlas-guided brain segmentation, statistical registration. MICCAI, pages 26-37, 2007. 1 